\title{
General Relativity and Gravitation introduces Consecutive Article Publishing
}

\author{
Ramon Khanna · Abhay Ashtekar • Roy Maartens
}

Published online: 10 December 2013

(C) Springer Science+Business Media New York 2013

Bibliographic information of articles published in physics and astronomy journals is changing rapidly towards a system in which articles are referenced individually rather than using page numbering within issues and volumes of a given journal. This way any delay in assigning the final citation information to an article is avoided.

Beginning with Volume 46 (2014) the journal General Relativity and Gravitation adopts such a system. Instead of a continuous pagination of articles throughout a volume, each article will have an internal pagination beginning with page 1 . The identification of an article within a volume by its first page number will be replaced by identification by an article citation-id. The bibliographic information (the "reference") shown in the header on the first page of an article will be built as follows:

Gen Relativ Gravit ([publication year]) [volume number]:[article citation-id] DOI [DOI]

(an example is the bibliographic information in the header of this editorial).

With this change papers will be available with their full and final bibliographical information as soon as they are published online and will fill up the currently open issue until its closure date. The journal will therefore stop publishing articles in Springer's

R. Khanna $(\varangle)$

Springer, Heidelberg, Germany

e-mail: Ramon.Khanna@springer.com

\author{
A. Ashtekar \\ Institute for Gravitation and the Cosmos, University Park, PA, USA \\ R. Maartens \\ University of Western Cape, Cape Town, South Africa \\ R. Maartens \\ University of Portsmouth, Portsmouth, UK
}


Online First ${ }^{\mathrm{TM}}$ modus. The time from acceptance to online publication of an article is not affected by this change. The final citation of an article will be of the form:

Khanna, R., Ashtekar, A., Maartens, R.: General Relativity and Gravitation introduces Consecutive Article Publishing. Gen. Relativ. Gravit. 46, 1612 (2014)

We believe this change will make a step towards a simpler system of bibliographic information within the framework of short online publication times of individual articles. 